Aim of the study: The arsenal of questions and answers about the minor cancer initiating cancer stem cell (CSC) population put responsible for cancer invasiveness and metastases, has left with an unsolved puzzle. Specific aims of a complex project were partly focused on revealing new biomarkers of cancer. We designed and set up novel techniques to facilitate the detection of cancerous cells.

Materials and methods: As a novel approach, we investigated $B$ cells infiltrating breast carcinomas and melanomas (TIL-B) in terms of their tumour antigen binding potential. By developing the TIL-B phage display technology we provide here a new technology for the specific detection of highly tumour-associated antigens. Single chain Fv (scFv) antibody fragment phage ELISA, immunofluorescence (IF) FACS analysis, chamber slide technique with IF confocal laser microscopy and immunohistochemistry (IHC) in paraffin-embedded tissue sections were set up and standardized.

Results: We showed strong tumour-associated disialylated glycosphingolipid expression levels on various cancer cells using scFv antibody fragments, generated previously by uniquely invasive breast carcinoma TIL-B phage display library technology. Conclusions: We report herein a novel strategy to obtain antibody fragments of human origin that recognise tumour-associated ganglioside antigens. Our investigations have the power to detect privileged molecules in cancer progression, invasiveness, and metastases. The technical achievements of this study are being harnessed for early diagnostics and effective cancer therapeutics.

Key words: cancer stem cells, malignant melanomas, technical developments, tumour immunology.

Contemp Oncol (Pozn) 2018; 22 (1A): 41-47 DOI: https://doi.org/10.5114/wo.2018.73884

\section{Challenging tumour immunological techniques that help to track cancer stem cells in malignant melanomas and other solid tumours}

\author{
Beatrix Kotlan ${ }^{1}$, Vanda Plotar ${ }^{2}$, Klara Eles ${ }^{2}$, Szabolcs Horvath², \\ Timea Balatoni ${ }^{3}$, Orsolya Csuka ${ }^{4}$, Mihaly Újhelyi ${ }^{5}$, Ákos Sávolt ${ }^{5}$, \\ Andras Szollar ${ }^{5}$, Istvan Vamosi-Nagy ${ }^{5}$, Laszlo Toth ${ }^{5}$, Emil Farkas ${ }^{5}$, \\ Jozsef Toth$^{2}$, Miklos Kasler ${ }^{6}$, Gabriella Liszkay ${ }^{3}$
}

\author{
${ }^{1}$ Molecular Immunology and Toxicology, National Institute of Oncology, Budapest, \\ Hungary \\ ${ }^{2}$ Center of Surgical and Molecular Pathology, National Institute of Oncology, Budapest, \\ Hungary \\ ${ }^{3}$ Department of Pathogenetics, National Institute of Oncology, Budapest, Hungary \\ ${ }^{4}$ Oncodermatology Department, National Institute of Oncology, Budapest, Hungary \\ ${ }^{5}$ Center of Oncosurgery, National Institute of Oncology, Budapest, Hungary \\ ${ }^{6}$ Board of Directorship, National Institute of Oncology, Budapest, Hungary
}

\section{Introduction}

Identifying cancer initiating cancer stem cells (CSC) has been a challenging task for many years. Even more so because the cancer stem cell concept has been awaiting its approval for decades. There can be no doubt that extensive experimental efforts and intensive technical improvements have led to a better understanding of this minor cancer cell population. Thanks to the various high throughput analyses to reveal mechanisms that drive cancer stemness and the characteristics of cancer stem-like and cancer stem cells hidden in the cancerous tissue microenvironment, there are important findings on drug resistance and cancer metastatic process. All previous and present relevant investigations help to solve the "CSC puzzle". The CSC story goes back to the first relevant studies with acute myeloid leukaemia (AML) [1], after which many other reports were announced showing important relevant data in solid tumours [2,3]. Because this minor cancer initiating cell (CIC) population was put responsible for metastatic processes and refraction to conventional cancer therapeutics [4], there is a special need to solve the problems in this topic. Substantial information on strategies identifying and selectively targeting CSCS would have the capacity to drive the cancer research field towards entirely new targeted cancer therapeutics that could be more effective and less toxic.

There are questions in highlights, whether certain biomarkers could eventually be found to characterise this minor cell population or follow in real time any ongoing changes at cellular or molecular levels? CSCs are endowed with the capacity to snake through the immunological supervision control, and this trick is still hidden despite the recent efforts [5-7]. We can only speculate, which of the revealed cell surface structures, molecular biological markers, or functional parameters could be selected as main champions in the arena of metastatic cancer elimination approaches? Functional genomics became the number one technique to serve with information on cancer stemness, while showing a new way to follow the cancer metastatic process [8, 9].

We approached the problem with another theory. Taking into account that „Nature must know the answer to cancer”, we have developed a novel strategy: our molecular genetic technology [10, 11], essential findings on 
cancer associated antigens [12] and a complex tumorimmunological project [13] helped us to reveal cancer targeting molecules. B lymphocites accumulated in the tumor microenvironment, the so called tumor infiltrating B cells (TIL-B) that can be found in breast cancers and other solid tumors $[14,15]$, can be analysed in terms of their antibody repertoire. These pioneering results could be corroborated in malignant melanomas [16]. We describe here a complex protocol by harnessing immune B cells from the tumor microenvironment to reveal tumorassociated antigens (TAA).

\section{Materials and methods}

\section{Patients}

Tissue samples of patients with nodular melanoma (NM) and superficial spreading melanoma (SSM) and frozen tissue specimen of one patient with invasive ductal breast carcinoma were gathered. Ethical permission (No: ETT TUKEB 15462-02/2010) and the patients' formal consent was provided.

\section{Cell lines}

Melanoma (SK-Mel28) and invasive ductal breast carcinoma (MDA-MD 231) cell lines purchased from American Type Cell Collections (ATCC) were maintained in steadystate culture conditions, using RPMI-1640 or DMEM (Sigma-Aldrich, San Diego, CA) supplemented with penicillin, streptomycin, gentamycin, amphotericin B and 5\% FBS (Sigma) in $T 25 \mathrm{~cm}^{2}$ or $T 75 \mathrm{~cm}^{2}$ flasks or chambers. Confluent cultures were harvested by EDTA $0.02 \%$ or Trypsin/ EDTA (Sigma) and checked for viability (by Trypan Blue $(0.4 \%)$ exclusion) and tested by indirect immunofluorescence (IF) assay.

\section{TIL-B antibody phage display}

cDNA was reverse-transcribed from RNA preparations of B cells in the tumor, using (RNeasy Qiagen and Total RNA isolation Kit Nucleon Spin RNA II, Nucleon Spin RNA L, Macherey - Nagel, Germany) or standard method with column purification (RNeasy MinElute Cleanup, Qiagen Germany). Antibody variable region genes were amplified with our previously developed technique and single chain (scFv) antibody variable region genes were constructed from TIL-B Ig VH and TIL-B Ig Vk gene regions. We used various molecular biological and biotechnological processes described in our original protocols for ScFvk phage display library generation $[12,14]$. Some further technology developments are summarised in our upcoming papers.

\section{ScFv Phage ELISA and soluble scFv ELISA}

We tested the antibody fragments of the original ScFv phage display library in ELISA, against "native" tumor cell membrane preparations, coated to 96 well microtiter plates. ScFv molecules with specific binding capacity to invasive ductal breast carcinoma and melanoma cells were selected. Recombinant Phage Antibody System (RPAS), scFv Expression and Detection Module (Amersham Biosciences) and pET26b vector-based expression systems
(Novagen) were used. The produced soluble scFv fractions of interest were validated quantitatively and qualitatively.

\section{Defining tumorassociated antigen binder molecules}

Coding regions of selected tumor binder scFv antibody fragments were subjects to novel detection and expression systems, using anti-E Tag, anti-c-myc or anti-His tag systems. Native membrane preparations and "membrane ghosts" of invasive ductal breast carcinoma and malignant melanoma cells and purified target molecules of interest were used in dot blot.

\section{Immunohistochemistry (IHC)}

Formalin-fixed, paraffin-embedded primary and metastatic tissue sections were deparaffinized in Xilol and Ethanol, then endogen hydrogene peroxidase was blocked and antigen retrieval was performed by heat-exposure using Microwave (Meditest MFX800-3). After blocking with 3\% bovine serum albumin (BSA) in PBS, tumorassociated and other IHC panel antigen specific monoclonal antibodies were reacted on the slides $\left(4^{\circ} \mathrm{C}\right.$, overnight or $\left.37^{\circ} \mathrm{C}, 30 \mathrm{~min}\right)$. Disialylated glycosphingolipid-specific antibodies (GD3, OacGD3) (Abcam, London, UK, Calbiochem and Axxora/ Alexis) and GSL antibody fragments were tested. AntiCD20 and antiCD19 (DAKO) were used for B cell detection. IHC was performed with Supersensitive TM One Step Polymer IHC Detection Kit System (BioGenex), using ImmPact TM AEC (3-amino-9-ethylcarbazole) substrate-diluent kit or 3,3'-Diaminobenzidine (DAB) substrate Kit (Vector, Burlingame, CA).

\section{Immunofluorescence Flow Cytometry - FACS Analysis}

Cancerous cell suspensions were reacted with unique GD3 ganglioside-specific antibodies (Abcam, London, UK), Calbiochem) or soluble fractions of expressed antibody fragments. We used suitable tag specific detection antibodies (anti-E Tag antibody (Abcam, London) and c-Myc monoclonal antibody (9E10) (ThermoFischer Sciuentific, Waltham, Massachusetts)) and Fluorescein isothiocyanate (FITC) or Phycoerythrin (PE) labelled goat anti mouse IgG (F(ab')2 fragment antibodies (R\&D systems / Bio-Techne Ltd, Abingdon) in our indirect IF assay. Flow cytometry FACS analysis was performed by CyFlow SL-Green, FloMax, Partec, Munster, Germany.

\section{Immunofluorescence - Confocal laser microscopy}

Fresh frozen cancerous melanoma tissues sections (6-8 $\mathrm{mm}$ ) were cut under tissue freezing media (Bio-Optika, Milano, Italy) by Slee Cryostat mnt (Auroscience), and were fixed by 4\% paraformaldehyde (PFA) PBS for 15 minutes. Chamber slide (LAB-TEK Brand Products, Nalgen Nunc, Naperville, US) cultures were blocked with 3\% BSA PBS, before tumor-associated disialylated glycosphingolipid-specific antibodies and the selected antibody fragments were added and reacted at $4^{\circ} \mathrm{C}$ overnight. Immunofluorescence labelling using second antibodies, was detected in confo- 

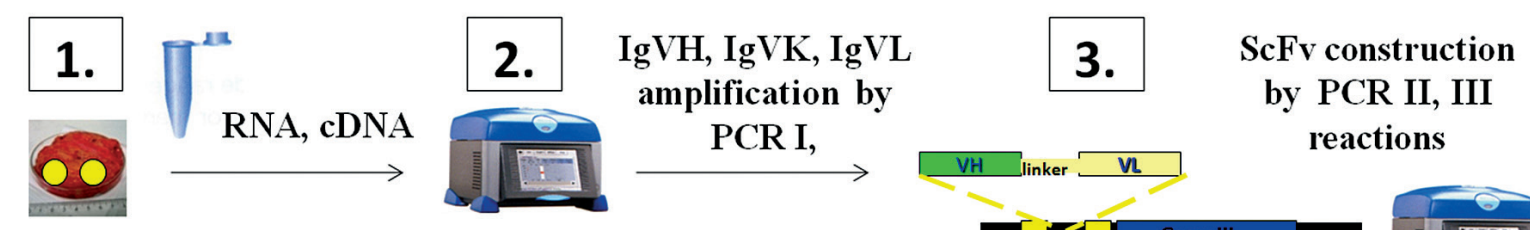
Cancerous tissue
with TIL-B
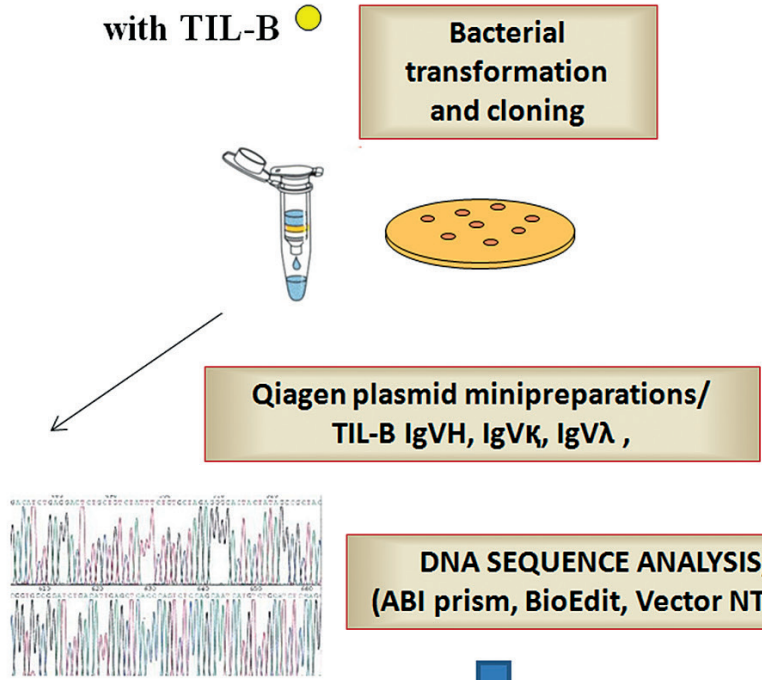

DNA SEQUENCE ANALYSIS/ (ABI prism, BioEdit, Vector NTI 11,

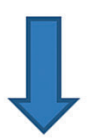

Defining overrepresented TIL-B immunoglobulin variable region genes

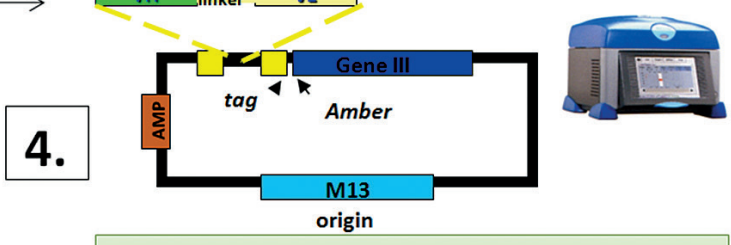

Insertion of scFv DNA into phagemid, bacterial transformation, TIL-B scFv PHAGE DISPLAY library

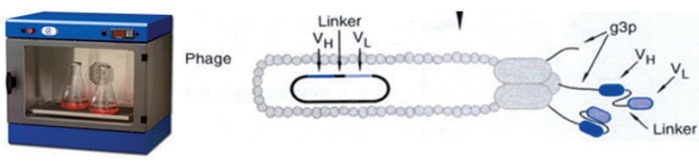

Selection and screening of scFv PHAGE DISPLAY library by phage and soluble ScFv ELISA

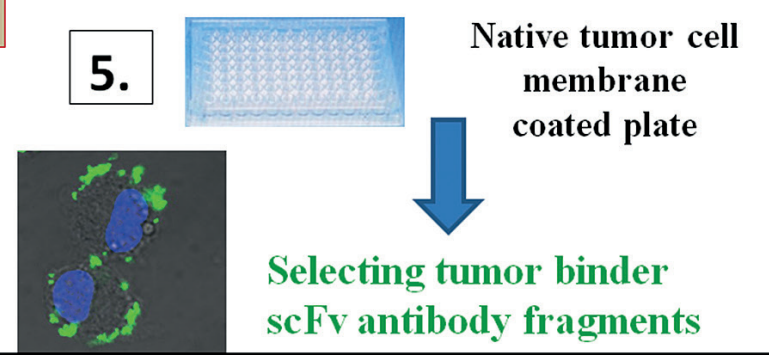

Fig 1. Flow chart of the novel tumor infiltrating B lymphocyte (TIL-B) scFv phage display protocol, suitable to obtain tumor antigen specific antibody fragments

cal laser microscopy (Nikon Eclipse E600, Nikon Model C1Lu3, Tokyo, Japan) or conventional IF microscopy.

\section{Results}

The solution within: Cancer specific antibody fragments provided by the tumor tissue

\section{Potential pathway for defining cancer antigens}

A complex protocol, based on the immune B cells within the tumor microenvironment, composed of consecutive cellular immunological assays, molecular genetic techniques and biotechnological processes turned out to be a potential pathway for revealing novel cancer antigens. We found that the TIL-B scFv phage display protocol is a suitable technique to obtaine tumor binder antibody fragments from $B$ cells, accumulated in any solid tumor (Fig. 1).

2. Detection of $B$ lymphocytes in the tumor microenvironment

Immunohistochemistry with CD20, CD19 specific antibodies showed a great variability of B lymphocyte infiltration in different breast carcinomas. B cells were represented from rare to abundant amounts in special types of breast carcinomas. We found that certain types of invasive ductal breast carcinomas show extremely high immune cell infiltration, and especially the B lymphocyte content is elevated (Fig. 2).

3. Cancer specific antibody fragments can be found in the TIL-B cell antibody repertoire

The TIL-B scFv phage display protocol and comparative DNA sequence analysis provided us with scFv coding DNA sequences of cancer specific binder antibody variable region genes. These scFv coding DNA sequences could be expressed as soluble scFv antibody fragments with an E-Tag or C-myc tag. These modifications enabled to test intact breast cancer and melanoma cells via indirect IF assay and FACS analysis. We defined strong IF labelling with our tumor binder anti disialylated glycosphingolipid antibody fragments (Fig. 3).

4. Detection of highly tumor-associated disialylated glycosphingolipids

Our previous soluble scFv ELISA and Dot blot techniques, using purified antigens and membrane preparations were compared to the present disialylated glycosphingolipid (GD3 ganglioside) detecting systems. Chamber slide cultured SK-mel 28 melanoma cells that strongly express GD3 disialylated GSLs showed intensive IF labelling with our se- 

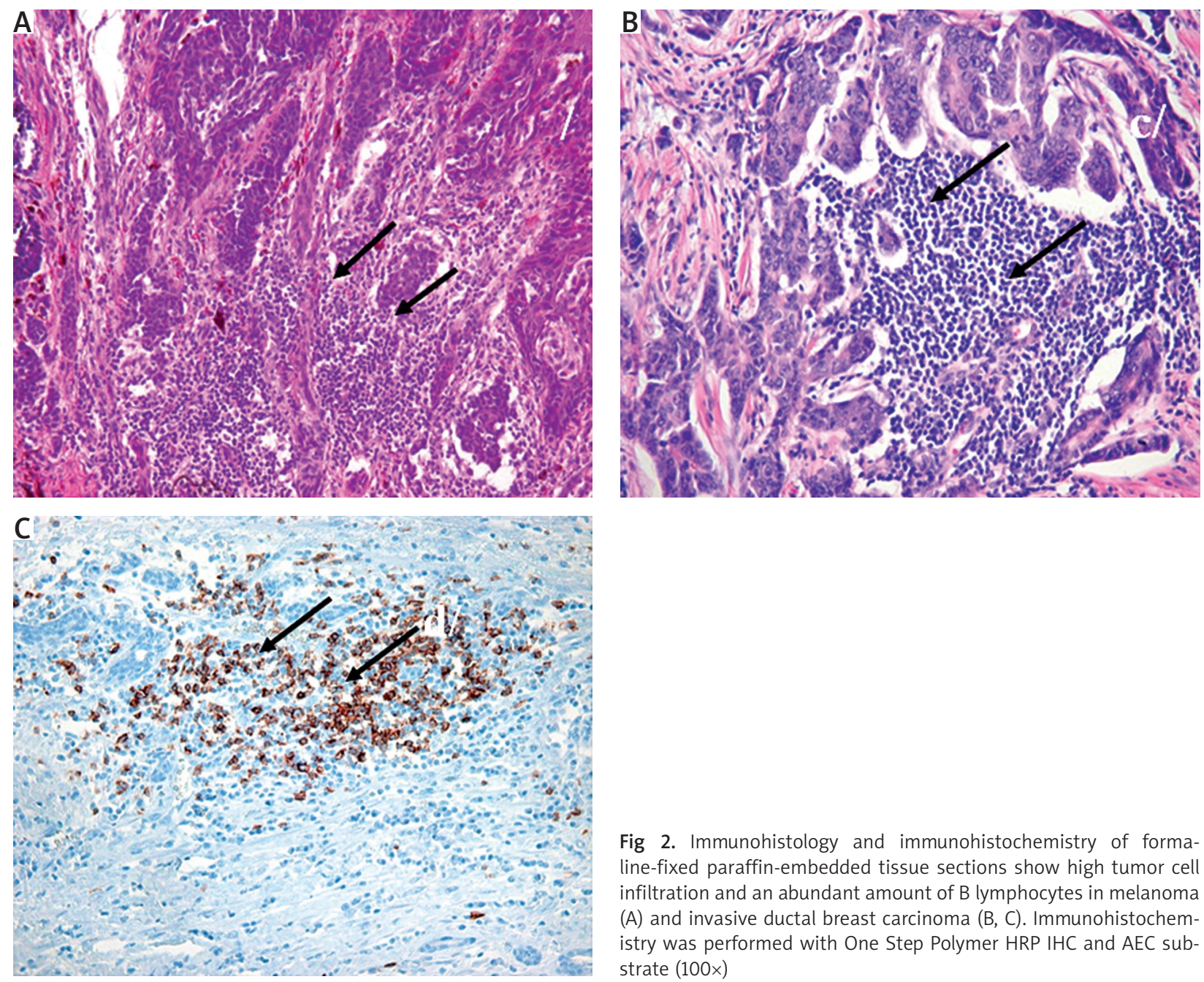

Fig 2. Immunohistology and immunohistochemistry of formaline-fixed paraffin-embedded tissue sections show high tumor cell infiltration and an abundant amount of $B$ lymphocytes in melanoma (A) and invasive ductal breast carcinoma (B, C). Immunohistochemistry was performed with One Step Polymer HRP IHC and AEC substrate $(100 x)$

lected scFv molecule in an indirect IF confocal laser microscopy detection system (Fig. 4). The frozen tissue sections of two malignant melanoma patients' surgical specimen gave intensive expression of GSL structures by immunohistochemistry (Fig. 5).

\section{Discussion}

We describe here a novel theory-based approach, where new technologies were introduced. These investigations finally lead to tumor-immunological findings with new cancer diagnostic and therapeutic impacts. According to the original hypothesis, the tumor holds the "key solution" for its fate. Immune B cells in the tumor microenvironment provide essential antibody variable gene regions with cancer membrane structure revealing capacity. Our complex TIL-B phage display analysis, the phage and soluble scFv ELISA techniques have the potential to reveal novel cancer associated antigens. Our previous and present studies strengthen the theory about obtaining strongly tumor-associated disialylated glycosphingolipid-specific human scFv antibody fragments of human origin. The whole process could serve as a gateway technology to gain specific, human antibody fragments targeting GD3 gangliosides, other GSLs and their derivatives.
Using the tumor infiltrating B lymphocyte population found in the cancerous tissue in the tumor microenvironment turned out to be a challenging idea. Our comprehensive study with carefully designed protocol and the technical improvements helped to understand more questions about the TIL-B cells. The sensitive techniques in the tumor immunological, molecular genetic and biotechnological processes lead to reveal essential cancer binder antibody fragments with unique specificities to tumor-associated disialylated glycosphingolipids. Our original model system was based on a rare type of invasive ductal breast carcinomas, showing extensive B cell infiltration and other specific characteristics $[17,18]$. Additional relevant information on the antibody repertoire of patients with breast cancer [19] or malignant melanomas [13] supported the further potentials of this approach. Comparative DNA sequence analyses seem to be an effective effort helping to reveal human antibody variable region genes of interest. We state that a theoretical comparative DNA analysis parallel with an experimental testing of the specific tumor binding capacity is an effective way to define highly cancer associated antigen-specific antibodies. We may conclude that valuable information about characteristic tumor-associated glycolipid and glycoprotein structures on the cancerous 

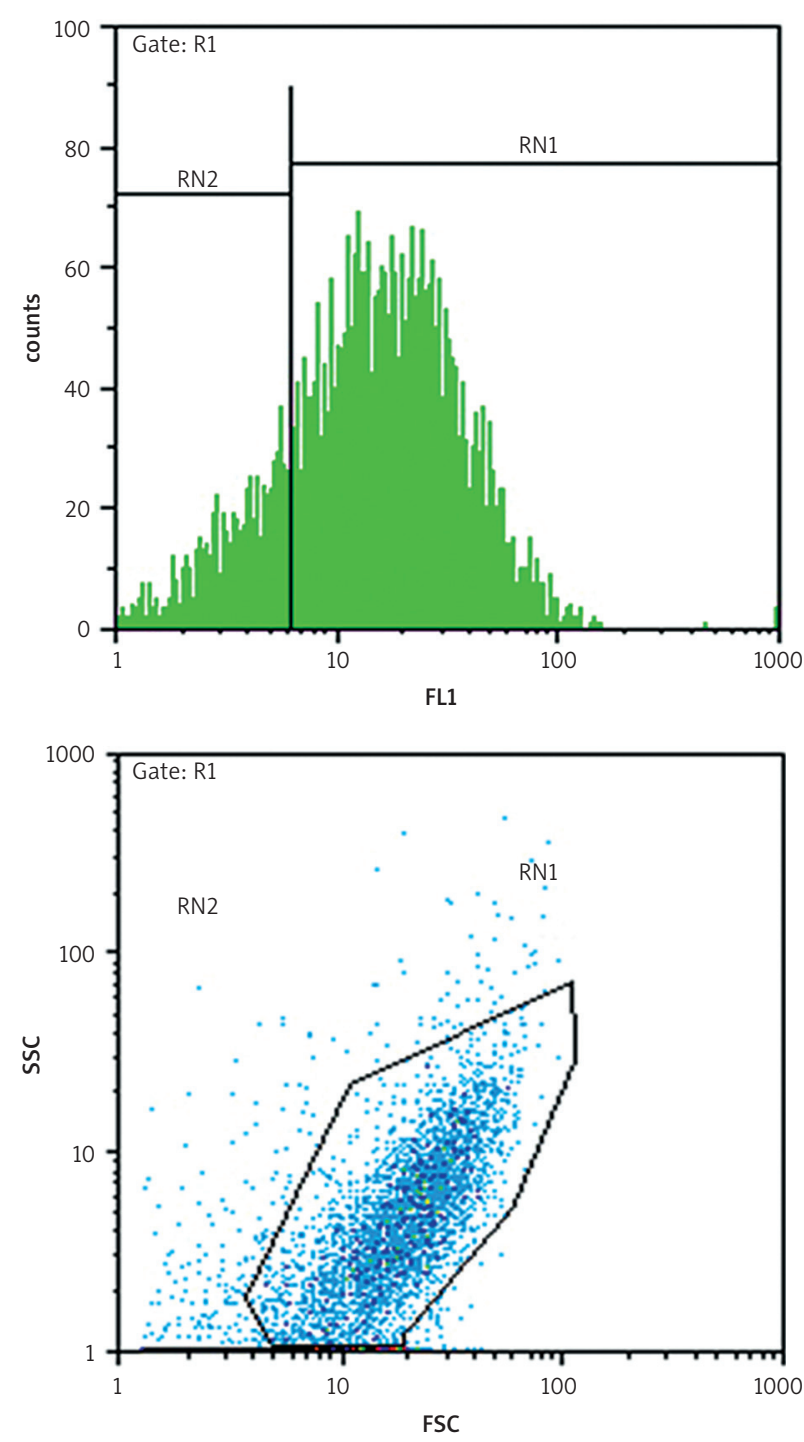

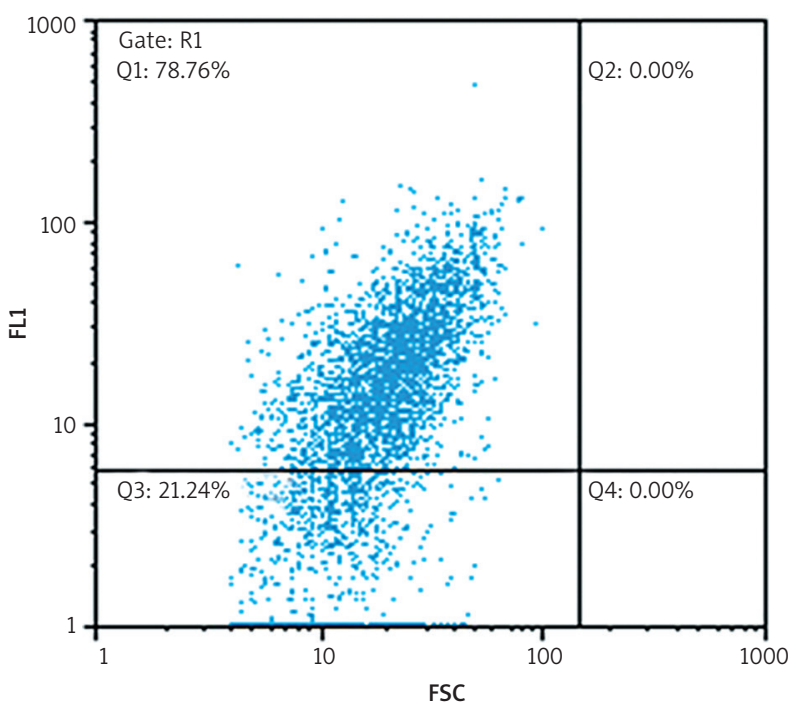

Fig 3. Immunofluorescence - FACS analysis evidenced strong expression of unique sialilated GD3 glycosphingolipids on the initiated melanoma cell lines with the soluble scFv antibody fragment in a suitable immunofluorescence labelling reaction

cells may be obtained with the "help of TIL-B cells". The presented complex TIL-B phage display strategy is a powerful technology to obtain human antibody fragments and testing specific tumor binding characteristics.

We found that our TIL-B cell-originated soluble ScFV-S were an excellent tool to detect glycosphingolipid-based tumor antigens on various cancer cells. However, choosing the suitable label tag (E-Tag or C-myc coded labelling) essentially depends on the selected reaction systems. Technology varies according to whether cultured cells are investigated in suspension or in adherent forms. We could show that the soluble scFv tumor binder antibody fragment preparations retained their tumor-associated antigen binder capacity. Cells in chamber tissue culture as well as in the frozen tissue criostat sections could well preserve the glycolitic sites' antigenicity. Reaction conditions and the paraformaldehyde fixation technique we used help to standardise the circumstances of the challenging detection of "tricky" sugar- and lipid-based membrane structures.

Our previous soluble scFv ELISA and Dot blot techniques, using purified antigens and membrane preparations were compared to the present disialylated glycosphingolipid detecting systems. The selected TIL-B-originated antibody
Fig 4. Detection of highly tumor associated disialylated glycosphingolipids on melanoma cells in a chamber slide culture system by immunofluorescence confocal laser microscopy 

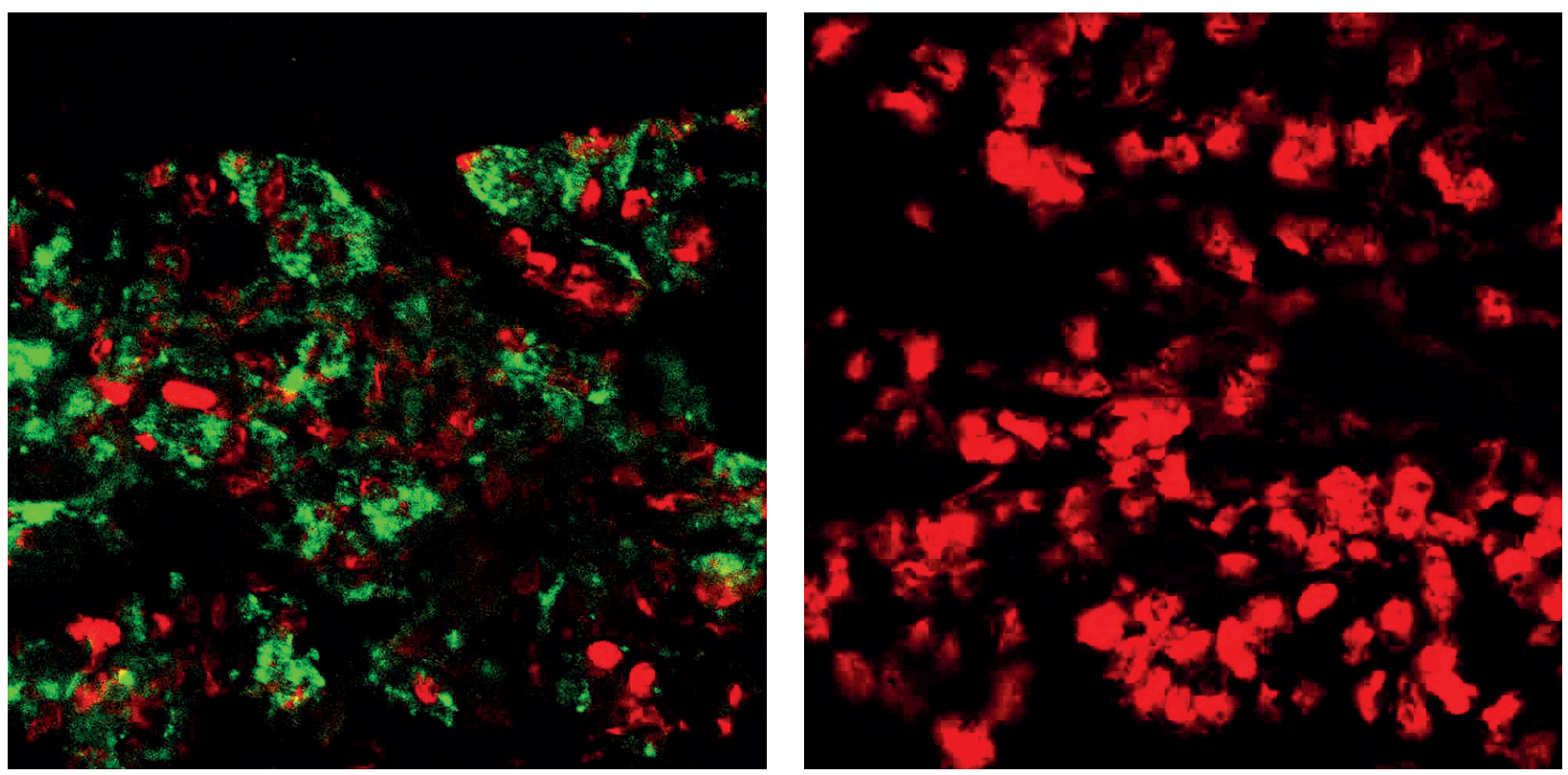

Fig 5. Unique GD3 ganglioside tumor associated antigen expression was shown on metastatic melanoma tissue cryostat sections with FITC conjugated labelling antibody and propidium iodide nucleus staining

fragments are useful tools for immunohistochemistry with fresh frozen cancerous tissue sections. Additional technical improvements are needed for these antibody fragments to meet the requirements necessary for IHC in paraffin-embedded tissue sections. Identifying the targeted disialylated glycosphingolipid (GD3 ganglioside, OAC GD3 ganglioside) and related other glycosphingolipids (GSLS) on various solid tumors [20-22] can be considered as an important milestone on the "road" to define useful cancer biomarkers [23, 24].

By gathering new scientific information on glycosylated antigenic sites, the large family of glycosphingolipids and the broad functional importance of these structures the growing field of glycoimmunomics has gained an utmost momentum. There is a slowly but straightforwardly accumulating evidence of these molecules in cancerogenesis, cancer proliferation and metastases [25]. Aberrant glycosylation expressed in glycosphingolipids and glycoproteins in tumor cells have been implicated as an essential mechanism in defining stage, direction and fate of tumor progression [26]. A rapidly evolving biological system involves sialic acids, a family of monosaccharides that are terminal caps on cell surface and secreted molecules of vertebrates, and play multifarious roles in immunity [27]. There are multiple human-specific evolutionary changes in inhibitory and activating Siglecs, immune cell receptors that recognize sialic acids as "self-associated molecular patterns" (SAMPs) to modulate immunity $[27,28]$. Important previous and recent findings evidence the fact that the detection of various glycosphingolipid-specific human antibodies have diagnostic value [29] and should be harnessed for new therapeutic strategies. All this is in line with our recent findings on a new technology to reveal cancer stem cells [16, 30].

Results of a detailed immunoglobulin repertoire analysis made it feasible to select overrepresented heavy and light chain immunoglobulin variable gene regions. Selected tumor-specific antibody fragment coding DNA sequences are excellent tools for further antibody engineering technologies [31, 32]. It has been shown that scFv, Fab fragments or diabodies, tetrabodies etc have important beneficial features for therapeutic usage [33]. Tumor-specific scFv antibody fragment coding DNA sequences can be subjects to next genetic engineering issues, like building a chimeric antigen receptor (CAR) to add new tumor antigen binding sites onto immune T cells $[34,35]$. The presented data hold the promise to develop genetically modified immune $T$ cells to redirect and eliminate cancer stem cells.

The Grants: Harry J. Lloyd Charitable Trust (HILCT) Melanoma Research Award/2010, Fulbright Grant No: $1214104 / 2014$ and Hungarian OTKA T048933 given to BK are acknowledged.

The authors declare no conflict of interest.

\section{References}

1. Bonnet D, Dick JE. J. Human acute myeloid leukemia is organized as a hierarchy that originates from a primitive hematopoietic cell. Nat Med 1997; 3: 730-7.

2. Singh SK, Clarke ID, Terasaki M, Bonn VE, Hawkins C, Squire J, Dirks PB. Identification of a cancer stem cell in human brain tumors. Cancer Res 2003; 63: 5821-8

3. Schmidt P, Kopecky C, Hombach A, Zigrino P, Mauch C, Abken H. Eradication of melanomas by targeted elimination of a minor subset of tumor cells. Proc Natl Acad Sci U S A 2011; 108: 2474-9.

4. Kim YJ, Siegler EL, Siriwon N, Wang P. Therapeutic strategies for tageting cancer stem cells. I Cancer Metastasis and Treatment 2016: 2: 233-42.

5. Trautmann F, Cojoc M, Kurth I, Melin N, Bouchez LC, Dubrovska A, Peitzsch C. CXCR4 as Biomarker for Radioresistant Cancer Stem Cells. Int J Radiat Biol 2014; 90: 668-99

6. Qual D, Joyca JA. Microenvironmental regulation of tumor progression and metastasis. Nat Med 2013; 19: 1423-37. 
7. Lu R, Turan T, Samayoa J and Marincola FM. Cancer immune resistance: can theories converge? Emerging Topics in Life Sciences 2017; 1: 411-9.

8. Dohertya MR, Cheonb H, Junka DJ, Vinayakc S, Varadanc V, Tellig ML, Fordg JM, Starkb GR, Jackson MW. Interferon-beta represses cancer stem cell properties in triple-negative breast cancer. Proc Natl Acad Sci U S A 2017; 114: 13792-7.

9. Bleau AM, Hambardzumyan D, Ozawa T, Fomchenko El, Huse JT, Brennan CW, Holland EC. PTEN/PI3K/Akt pathway regulates the side population phenotype and ABCG2 activity in glioma tumor stem-like cells. Cell Stem Cell 2009; 4: 226-35.

10. Kotlan B, Gruel N, Zafrani B, Furedi G, Foldi J, Petranyi Gy G, Frid man WH, Teillaud JL. Immunoglobulin variable regions usage by B-lymphocates infiltrating a human breast medullary carcinoma. Immunology Letters 1999; 65: 143-51.

11. Kotlan B, Simsa P, Gruel N, Foldi J, Fridman WH, Petranyi Gy, Teillaud $J$ L. A scFv phage display mini library generated from the immunoglobulin repertoire of breast medullary carcinoma infiltrating B lymphocytes. Disease Markers 2000; 16: 25-7.

12. Kotlan B, Simsa P, Teillaud JL, Fridman WH, Toth J, McKnight M, Glassy M. Novel ganglioside antigen identified by B cells in human medullary breast carcinomas. The proof of principle concerning the infiltrating B lymphocytes. J. Immunol 2005; 175: 2278-85.

13. Kotlan B, Liszkay G, Blank M, et al. The novel panel assay to de fine tumor-associated antigen-binding antibodies in patients with metastatic melanomas may have diagnostic value. Immunol Res 2015; 61: 11-23.

14. Gruel N, Kotlan B, Zafrani B, Teillaud JL. Generation of scFv from a phage display mini-library derived from tumor-infiltrating $B$ cells. In: RT-PCR Protocols/ Methods in Molecular Biology. O'Connel J (ed.). The Humana Press, Inc, Totowa, New Jersey USA, 2002; 193: 281-300

15. Coronella JA, Spier C, Welch M, Trevor KT, Stopeck AT, Villar H, Hersh EM. Antigen-driven oligoclonal expansion of tumor-infiltrating B cells in infiltrating ductal carcinoma of the breast. J Immunol 2002; 169: 1829-36.

16. Kotlan B, Liszkay G, Naszados Gy, et al. Minor tumor infiltrating B cells opened a door to reveal and eliminate cancer initiating cells in metastatic melanoma. J Immunother Cancer 2014; 2: (suppl 1): p4.

17. Kouhen F, Benhmidou N, Afif M, et al. Prognosis of Medullary Carcinoma of the Breast: 10 years' Experience in a Single Institution. Breast J 2017; 23: 112-4

18. Kotlan B, Simsa P, Foldi J, Fridman WH, Glassy M, McKnight M, Teillaud JL. Immunoglobuline repertoire of B lymphocytes infiltrating human breast medullary carcinoma. Human Antibodies 2003; 12: 113-21.

19. Simsa P, Teillaud JL, Stott I D, Toth J, Kotlan B. Immunoglobulin variable region gene usage of $B$ lymphocytes in ductal breast carcinomas. Pathol Oncol Res 2005; 11: 92-7.

20. Kjeldsen TB, Clausen H, Hirohashi S, Ogawa T, Lijima H, Hakomori SI. Preparation and characterization of monoclonal antibodies di rected to the tumorassociated O-linked sialosyl-2-6 a-N-acetylgalactosaminyl (sialosyl-Tn) epitope. Cancer Res 1988; 48: 2214-20.

21. Chammas R, Sonnenburg JL, Watson NE, Tai T, Farquhar MG, Varki NM, Varki A. De-N-acetyl-gangliosides in humans: Unusual subcellular distribution of a novel tumor antigen. Cancer Res 1999; 59: 1337-46.

22. Ravindranath MH, Gonzales AM, Nishimoto K, Tam WY, Soh D, Morton DL. Immunology of gangliosides. Indian J Exp Biol 2000; 38: 301-12.

23. Masucci GV, Cesano A, Hawtin R, et al. Validation of biomarkers to predict response to immunotherapy in cancer: Volume I- pre-analytical and analytical validation. J Immunother Cancer 2016; 4: 76 100

24. Hendrickx W, Simeone I, Anjum S, et al. Identification of genetic determinants of breast cancer immune phenotypes by integrative genome-scale analysis. Oncolmmunology 2017; 6: doi.org/10.1080 /2162402X.2016.1253654

25. Pochechueva T, Jacob F, Fedier A and Heizelmann-Schwarz V. Tumor-Associated glycans and their role in gynecological cancers:
Accelerating translational research by novel high throughput approaches. Metabolites 2012; 2: 913-39.

26. Hakomori SI. Tumor malignancy defined by aberrant glycosilation and Sphingo(glyco)lipid metabolism. Cancer Res 1996; 56: 5309-18.

27. Varki A Are humans prone to autoimmunity? Implications fromevolutionary changes in hominin sialic acid biology. J Autoimmunity 2017; 83: 134-42.

28. Maverakis E, Kim K, Shimoda M, et al. Glycans in the immune system and The Altered Glycan Theory of Autoimmunity: A critical review J Autoimmunity 2015; 57: 1-13.

29. Ravindranath MH, Kelley MC, Jones RC, Amiri AA, Bauer BM, Morton DL. Ratio of IgG: IgM antibodies to sialyl Lewisx and GM3 correlates with tumor growth after immunization with melanoma-cell vaccine with different adjuvants in mice. Int J Cancer 1998; 75: 117-24.

30. Kotlan B, Balatoni T, Czirbesz K, et al. „Immune B Cells know it better": tumorimmunological panel assay to define tumor-associated antigen binding antibodies in patients with metastatic melanomas. J Transl Med 2015; 13: (Suppl 1): P16

31. Hudson PJ, Souriau C. Engineered antibodies. Nat Med 2003; 9: 129-34.

32. Teillaud JL. From whole monoclonal antibodies to single domain antibodies: think small Methods Mol Biol 2012; 911: 3-13.

33. Marshall MJE, Stopforth RJ, Cragg MS. Therapeutic antibodies: What have we learnt from targeting CD20 and where are we going? Front Immunol 2017; 8: 1245-66.

34. Kershaw MH, Westwood JA, Parker LL, et al. A phase I study on adoptive immunotherapy using gene-modified $T$ cells for ovarian cancer. Clin Cancer Res 2006; 12 (20 Pt 1): 6106-15.

35. Kotlan B, Csuka O, Tóth L, et al. Genetic information from tumor-infiltrating B lymphocytes as a driver tool ("GPS") for anti-tumor T cell CARs. Hungarian Oncol 2016; 60: 64-71.

\section{Address for correspondence}

\section{Beatrix Kotlan}

Molecular Immunology and Toxicology

National Institute of Oncology

Rath Gy Street 7-9

Budapest 1122, Hungary

tel. $+3612248600 /$ ext. 3294

e-mail: kotlanb@netscape.net, kotlanbea@gmail.com 